2016. ("Primary morbidity and mortality from tuberculosis of the population of the Amur Region in dynamics 100 percent thousand population)". Currently, the level of the incidence of tuberculosis (diagnosed for the first time in life) in the Amur region amounted to 110 in 2012, and in 2012 - 100.2, in 2014 - 85, in $2015-76.9$, in 2016 66.9 cases 100 percent thousand of the population. Analysis of the dynamics of incidence rates of tuberculosis in the Amur Region as a whole for 2012-2016. indicates a change in this indicator in the direction of decrease.

In 2012, the greatest number of cases was detected in the Progress settlement - 200, in the Konstantinovsky district - 193.8, and in the Mikhailovsky district - 162.2 cases 100 percent thousand population. In 2013, the prevalence rates in the Belogorsky district were 186.2, in the Romny region - 181.8, in the Bureysky district - 151.1 cases 100 percent thousand of the population. In 2014, in the Svobodnensky district-168.9, in the Konstantinovsky district - 140.6, in the Shimanovsky district - 137.9 cases 100 percent thousand of the population. In 2015, in the Bureysky district - 154.5, in the Magdagachinsky region - 125.6, in the Ivanovo region - 119.3 cases 100 percent thousand of the population. In 2016, the incidence of tuberculosis in the Shimanov district was 153.8, in the city of Shimanovsk - 127.9, in the Belogorsky district - 120.9 cases 100 percent thousand of the population.

The total TB mortality in 2012 was the highest in the Svobodnensky district and amounted to 109.6, in Progress - 69.2, in Bureysky district - 60.1 cases 100 percent thousand people. In 2013 the greatest number of deaths from tuberculosis was registered in Raichikhinsk - 75.8, in the Ivanovo region - 52.0, in the Romny region - 45.5 cases 100 percent thousand of the population. In 2014, Ivanovo region - 51.8, in the Bureysky district - 48.9, in the Oktyabrsky district - 42.1 cases 100 percent thousand of the population. In 2015, the greatest number of deaths from tuberculosis was registered in the Shimanovsky district - 56.6, in the Svobodnensky district 47.9, in the Romny region - 46.0 cases 100 percent thousand of the population. In 2016 in the Mikhailovsky district - 71.4, in Raichikhinsk - 53.4, in the Bureysky district - 28.3 cases 100 percent thousand of the population.

Most people with tuberculosis are aged 18-44 years. The maximum incidence of tuberculosis is 25-34 years for women and 35-44yearsformen. Thepeak ofmortality from tuberculosisisattheage of 35-44yearsamongwomenand45-54amongmen.

Conclusions. In the Amur Region, there is a stabilization of the epidemic situation of tuberculosis with a tendency to decrease. The reduction of tuberculosis incidence and mortality from this disease occurs against the background of increased coverage of the population with preventive examinations for tuberculosis, development and implementation of modern clinical protocols, procedures and standards of medical care that take international experience into account and reduce the proportion of advanced forms of tuberculosis among newly diagnosed tuberculosis patients.

\title{
References
}

1. Laushkina, Zh. A. Hyperdiagnosis of tuberculosis in patients with malignant neoplasms of

the lungs/ Zh. A. Laushkina PN Filimonov // Tuberculosis and lung diseases. - 2014. - No. 5. - P. 56-59.

2. New approaches to the diagnosis and treatment of tuberculosis and its drug-resistant forms / E.M. Skryagi-

na // Recipe. - 2013. - No. 1. - P. 85-98.

3. Tuberculosis in the Russian Federation. Analytical review of statistical indicators for tuberculosis used in the Russian Federation. - 2011. - P. 223.

4. The epidemiological situation of tuberculosis in Russia. Anti-tuberculosis measures. Information letter No. 7-5 / 210

"CNIIOIZ" of the Ministry of Health of Russia. - Moscow: 2014. - 61 p.

UDC 616.24-002.155 DOI 10.22448/AMJ.2017.3.134-135

\section{DIFFICULTIES OF DIFFERENTIAL DIAGNOSTICS OF INTERSTITIAL LUNGS DISEASES}

\author{
Kostrova I.V., Prikhodko O.B.
}

Amur state medical academy, Blagoveshchensk, Russian Federation

Abstracts. The hardest problem in practice of general practitioner and pulmonologist is the problem of differential diagnostics of interstitial lungs diseases. We introduce the case of cryptogenic organizing pneumonia with ways of diagnostics and treatment of that disease.

Key words: interstitial lungs diseases, differential diagnostics, cryptogenic organizing pneumonia.

The hardest problem in practice of general practitioner and pulmonologist is the problem of differential diagnostics of interstitial lungs diseases. The traditional triad of the most common causes of pulmonary infiltrates during over many years is the classical differential series. However, with the advent of modern diagnostic methods, diseases that have previously occurred under the guise of those listed above or have not been diagnosed at all, have become plural. There are big difficulties, when radiologist or morphologist meet cases that they have not seen before in their practice. In this case even obtaining a complete histological material does not guarantee a correct diagnosis.

Material and methods. The clinical analysis of the case of a 54-year-old patient was done. During the examination, one of the forms of idiopathic interstitial pneumonia was diagnosed - the cryptogenic organizing pneumonia. The patient came to the department at 08.07 with complaints of fever to subfebrile digits, shortness of breath with moderate physical exertion. From an anamnesis: she considers herself ill since June 14, when she noted a chill after a hypothermia, the temperature was raised to $38^{\circ} \mathrm{C}$, she was treated symptomatically, 24.06 a segmental pneumonia in the lower lobe on 
the right side was diagnosed on chest radiography; antibacterial therapy (ceftazidime, levofloxacin) has been prescribed. On a background of treatment the temperature has decreased to subfebrile. 06.07 at X-ray revealed a negative dynamics with the spread of infiltration to the lower parts of the left lung. She was hospitalized in pulmonology department. From the past diseases - genital herpes, chronic tonsillitis. Harmful habits, allergic reactions, professional harmfulness denies.

The patient's condition is satisfactory; Skin and mucous membranes are clean, of normal color. Respiratory rate 17 in min. Percussion sound over the lungs is pulmonary. Vesicular breathing, crepitus in the lower parts on both sides, more on the left. Blood pressure 100/60 mm/Hg Heart auscultation: tones are clear, rhythmic, heart rate -80 per min. The tongue is wet.

The abdomen is painless. Liver: $9 \times 8 \times 7 \mathrm{~cm}$, the edge of liver is even. The kidneys are not palpable. The lumbar region is painless on both sides.

In the clinical blood test there was an increase in ESR up to $60 \mathrm{~mm} / \mathrm{h}, \mathrm{CRP}-37.4 \mathrm{mg} / \mathrm{L}$, procalcitonin $<0.5 \mathrm{ng} / \mathrm{ml}$. Other parameters are within the normal range. The patient was examined by CT of thoracic organs. On a series of tomograms, in S8 - S10 on both sides determined uneven compaction of pulmonary tissue due to infiltrative changes, against which background bronchial lumens are traced. A wide base of infiltration adjoins the pleura, and narrow - directed towards the root of the lung. Given the clinical picture, the progression of the disease against the background of antibacterial therapy, high density of infiltrates in the lungs, doubts were expressed regarding bacterial pneumonia.

Interstitial lung disease was more likely. 09.07 there was held video-thoracoscopy in the left, biopsy of the lower lobe of the left lung. Pathological conclusion: the lung tissue is deformed due to ingrowth of the respiratory bronchioles of the 1st order of granulation tissue in the form of polyps in the part of the alveoli cavity and in the part of the alveolar cavities - the accumulation of macrophages with an admixture of eosinophils and red blood cells with individual eosinophils, in other lumens alveolus is a fibrin accumulation with an admixture of eosinophils and neutrophils, the individual walls of the alveoli are thickened due to the small lymphocytic infiltration and proliferation of second type alveolocytes. Conclusion: obliterating respiratory bronchiolitis with organizing pneumonia. Arteriolar walls Fibroelastosis.

Based on the results of the examination, the patient was diagnosed with: Cryptogenic organizing pneumonia (obliterating bronchiolitis with organizing pneumonia). By this time, the patient's well-being improved - the temperature returned to normal, the dyspnea disappeared. Systemic corticosteroid therapy (prednisolone $25 \mathrm{mg} /$ day) has been started. In 2 weeks. there was further positive dynamics: no complaints, a decrease in ESR up to $42 \mathrm{~mm} / \mathrm{h}$, on CT of the chest - a decrease in the size and density of infiltrates in the lungs. In another 2 months. infiltration into lung tissue was no longer determined.

Idiopathic interstitial pneumonia combines several diseases from the group of interstitial lung diseases characterized by many similar features. Modern classification of ATS / ERS includes seven forms of IIP. The morphological substrate of COP is obliterative bronchiolitis with organizing pneumonia, characterized by the organization of exudate and fibroblastic reaction in the form of granulation tissue ingrowth into respiratory bronchioles, alveolar courses and alveoli.

The COP included in the classification of in respect of its idiopathic nature and similar to other forms of IIP, especially if the IIP progressestofibrosis. ACOPusuallyhasasubacutecourse with a relativelyshortperiod beforediagnosis(average 3 months), with a cough and shortness of breath of varying severity. The most common causes of OBOP are infection, drugs, radiation therapy and diseases of the connective tissue. If the cause is not obvious, OBOP called cryptogenic organizing pneumonia.

Conclusions. The clinical pattern of a COP is very similar to bacterial pneumonia. The disease has acute or subacute beginning, accompanied by fever, cough, and appearance finely wheezing in the areas of infiltration. Characteristically by the development of dyspnea from the first days of the disease. The standard of treatment of COP is the prescription of systemic corticosteroid - prednisolone at a dose of $0.75-1.5 \mathrm{mg} / \mathrm{kg}$ daily for $1-3$ months. Despite the generally good response to steroid therapy, $30-50 \%$ of patients after discontinuation of hormonal therapy relapse occurring in a milder form. Fiveyears survival rate in this disease is $90 \%$. The COP is one of the variants of reaction of the lungs to an infectious factor and can occur heterogeneous in different patients, from complete resolution to progressive course and formation of fibrosis.

\title{
References
}

1. Avdeev S. N. Idiopathic interstitial pneumonias: features of clinical picture and treatment // Farmateka, 2009. No. 19.

2. Avdeev S. N. Different variants of the course of idiopathic pulmonary fibrosis: phenotypes and comorbid conditions // Practical pulmonology, 2016. No. 2. P. 37 - 45.

UDC 612-017.2:613.166/.9]616-003.96:577.118 DOI 10.22448/AMJ.2017.3.135-137

\section{APPLICATION PHYTOADAPTOGENS FOR THE CORRECTION OF PHYSICAL LOADS ON THE BODY UNDER TEMPERA- TURE STRESS}

\author{
Litovchenko E.A., Korshunova N.V.
}

Amur State Medical Academy, 95 Gor'kogo Str., Blagoveshchensk, 675000, Russian Federation

Abstract In the experimental conditions investigated the possibility of increasing the resistance of rats to physical influence in the conditions of use of a mixture of adaptogenic products (Hypericum perforatum and Rhodiola rosea) in cold and heat stress. 